\section{Appendicitis Ultrasound: Comparison Study of the Radiology Resident to the Technologist and Attending}

\section{Abstract}

Background and objects: Community hospitals and academic centers may not always have a radiologist or sonographer on site, and the responsibility of US performance after hours maybe left to a resident. The purpose of this study was to evaluate a growing concern at our institution that a higher numbers of pediatric CT scans were being conducted overnight for the evaluation of acute appendicitis, as a result of a perception that scans performed by residents were not as accurate as those performed by technologists and/or attending.

Materials and methods: 573 patients initially evaluated by ultrasound for appendicitis, from April 2008 to March 2014. Studies were distinguished by either resident or technologist and attending (TA) performing the procedure. The junior resident was in the first to third years of training with at least 8 weeks of US experience. Follow-up computed tomography and operative reports were reviewed to determine if appendicitis was later confirmed.

Results: Of 462 studies in the pediatric non-pregnant group, ages one to eighteen years, 261 (56.5\%) were performed by a junior resident and 201 (43.5\%) were performed by the TA group. The sensitivity of diagnosing acute appendicitis in the first ultrasound by the resident and TA groups were $59 \%$ and $67 \%$, respectively $(p=0.73)$. Additional testing ordered after a negative initial US was higher in the resident group than the TA group, 85 (39.5\%) and 46 (26.4\%), respectively $(p=0.010)$. The number of false negatives in the overall, resident and TA groups were $35(38 \%), 24(41 \%)$ and $11(33 \%)$, respectively $(p=0.68)$.

Conclusion: Although appendicitis US has been traditionally touted as operator dependent, there was no statistical difference in this study between residents and attendings of varying levels of experience. Additionally, more follow up studies were ordered when the initial study was negative and performed by the resident.

Keywords: Appendicitis; Attending; Comparison; Operator; Resident; Ultrasound

\section{Allaei A, Goldfisher R, Neimark $\mathrm{M}$ and Amodio J}

State University of New York, Downstate Medical Center, Brooklyn, New York, USA

Corresponding author: John Amodio

”john.amodio@downstate.edu

State University of New York Downstate Medical Center, Brooklyn, New York, USA.

Tel: 917-968-3283

Citation: Allaei A,Goldfisher R,Neimark M, et al. Appendicitis Ultrasound: Comparison Study of the Radiology Resident to the Technologist and Attending. J Pediatr Care. 2016, 2:1.

Received: February 23 , 2016; Accepted: March 30,2016 ; Published: April 8, 2016

\section{Introduction}

Appendicitis is a common disease entity with potentially serious morbidity and mortality affecting nearly 70,000 children in the United States each year [1]. Traditionally appendicitis was diagnosed primarily on clinical grounds; however no single history, physical or laboratory finding can definitively make the diagnosis. More importantly a delay in the diagnosis can result in increased risk of sepsis, obstruction, perforation, abscess, peritonitis and death [2].
Although computed tomography (CT) is the most accurate method of diagnosis and the preferred method in the adult population, ultrasound (US) is the preferred modality in the pediatric population given the radiation dose delivered by CT. The pediatric population is particularly sensitive to ionizing radiation and a growing concern is the increasing use of $C T$ on pediatric patients in emergency departments in United States [3].

Sonography provides a radiation-free method of diagnosing appendicitis in pediatric patients. Appendicitis on US can be seen 
as a fluid filled, non-compressible bind-ending tubular structure, and traditionally with a diameter of more than $6 \mathrm{~mm}$ [4]. However, sonography is a dynamic study that is operator-dependent and has its own pitfalls.

Community hospitals and academic centers may not always have a radiologist or sonographer on site, and the responsibility of US performance after hours maybe left to a resident. The purpose of this study, originally a quality and safety project, was to evaluate a growing concern at our institution that a higher numbers of pediatric CTs were being conducted overnight for the evaluation of acute appendicitis. To our knowledge this is the first study comparing the diagnostic accuracy of US performance by a junior radiology resident to the technologist and attending (TA), utilizing follow up cross sectional imaging and operative reports as the gold standard.

\section{Materials and Methods}

A retrospective analysis was undertaken of 573 patients who were initially evaluated by US for clinical suspicion of appendicitis, from April 2008 to March 2014. This data was abstracted from the electronic medical records, and radiologic data files and picture archiving and communication system (PACs) of Kings County Hospital, Brooklyn, New York. Our institution is a general large urban medical center that sees approximately 250 pediatric patients a day. This single-center retrospective study was approved by our institutional review board and compliant with the Health Insurance Portability and Accountability Act.

Studies were distinguished by either radiology resident or technologist and attending performing the procedure, age and sex of the patient, pregnancy status, and presence of follow up diagnostic studies. The junior resident was a resident in the first to third years of training and had received at least 8 weeks of hands on US rotation experience, 4 weeks in general ultrasound and 4 weeks as a part of pediatric radiology, with exposure to pediatric appendicitis protocol. The exact number of cases performed by each resident could not be quantified due to daily variability.

Resident reports were finalized the following morning by the covering attending with the images provided. Since the attending only had the provided images to confirm an impression, there was not an opportunity to re-scan the patient. Majority of the clinical decisions and follow up imaging would have already been performed within a 12-hour period. Studies performed during daytime hours by the technologist were re-scanned by the covering attending radiologist. Given that our institution is not a pediatric hospital, the technologists and attending's practice both pediatric and adult radiology.

Sonography was performed on a variety of machines; however the protocol was similar in both groups. Adequate scanning required visualization of all four quadrants and the site of pain with the use of a linear array probe ranging from 9-18 $\mathrm{MHz}$. At our institution CT examinations following sonography for acute appendicitis are performed with IV contrast and are acquired with a slice thickness of $0.625 \mathrm{~mm}$ from the second lumbar vertebrae to the pubic symphysis. If perforation or an abscess was suspected, the field of view would be extended to the level of the diaphragm, which is the routine appendicitis protocol for our institution.

A positive study was one where the final impression by the radiologist was documented as acute appendicitis or suggestive of appendicitis, with the diagnostic criteria of a non-compressible appendix larger than $6 \mathrm{~mm}$. Equivocal reports included findings of suspicious free fluid and/or an incompletely visualized appendix (Figure 1), with the final diagnosis resting on clinical findings or additional imaging studies.

Follow-up CT, operative and pathology reports were reviewed to determine if appendicitis was later confirmed. If the initial or follow up study was positive, CT and operative reports were also reviewed to determine the location of the appendix, and whether or not there was perforation. Statistical significance between the outcomes of the two groups was calculated based on the Fisher exact test.

\section{Results}

A total of 573 US appendicitis cases were found on PACS, of which, 462 studies were in the pediatric non-pregnant group, ages one to eighteen years. 261 (56.5\%) were performed by a junior resident and 201 (43.5\%) were performed by a technologist or attending (Table 1). The number of true positives in which the initial US

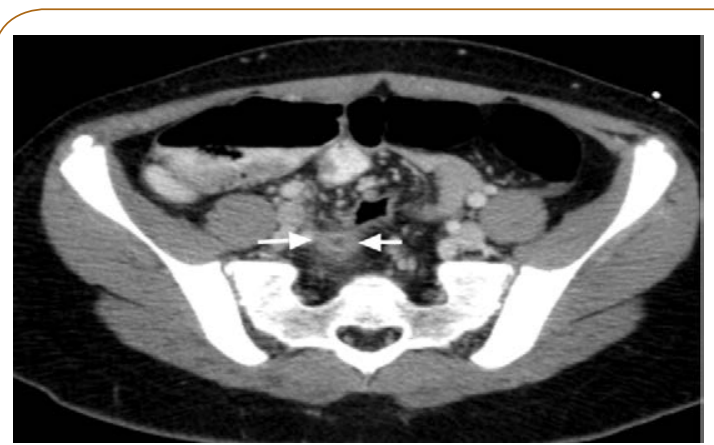

Figure 1a Equivocal Ultrasound of a non-perforated appendicitis. Ultrasound demonstrated an incompletely visualized appendix (+) in the right lower quadrant measuring $5 \mathrm{~mm}$ with suspicious appearing free fluid (not shown).

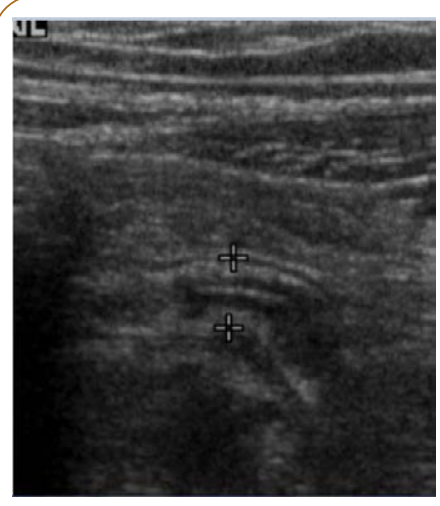

Figure 1b Correlate CT demonstrating a retrocecal appendix, measuring $5 \mathrm{~mm}$ and adjacent stranding (arrows) confirming appendicitis. 
correctly diagnosed appendicitis in the resident and TA groups were 34 and 22 , with a sensitivity of 0.57 and 0.67 , respectively $(p=0.73)$.

In the resident group, there was one false positive where the resident misinterpreted a tubular structure as the appendix, and a repeat US the following morning was found to be negative. Of the remaining 34 positive reports, five were confirmed by a follow up CT, one by US and the remainder by surgery (Table 2). Two of these cases were perforated and retrocecal, while a third was just retrocecal.

There were 216 initial negative US studies in the resident group, of which, 85 patients underwent additional follow up diagnostic imaging, the majority of these being CTs $n=81$, (Table 2). Twentyfour of these follow up studies were found to be positive for appendicitis (Table 2). An additional 2 in the equivocal category was also positive. There were overall 200 true negatives, prevalence of 0.23 and specificity of 0.995 . This was with the assumption that those without subsequent surgery or a follow up study had a normal appendix.

In the technologist and attending group, CT confirmed 6 of the 22 positive initial US cases, while the remainder was confirmed at surgery. Within this group, two appendices were retrocecal, one perforated, and one perforated and retrocecal. There were 174 initial negative US reports, of which, 46 underwent additional follow up diagnostic imaging, all of which were CTs. Eleven of these follow up studies were positive for appendicitis (Table 3 ). There was one additional acute appendicitis in the equivocal category giving overall 167 true negatives, prevalence of 0.17 and specificity of 1.

Overall, there were 85 (39.5\%) and 46 (26.4\%) additional studies ordered after a negative initial US in the resident and TA groups, respectively $(p=0.010)$. The number of false negatives in the overall, resident and TA groups were 35 of 91 (38\%), 24 of 58 $(41 \%)$, and 11 of $33(33 \%)$, respectively $(p=0.68)$.

Equivocal US reports accounted for 10 cases in the resident group and 5 in the TA group $(p=0.60)$. In the resident group, two patients underwent additional diagnostic evaluation with $\mathrm{CT}$ and were found to have appendicitis. One case was retrocecal, while the other was perforated and retrocecal. Of the 5 equivocal cases

Table 1 Comparison of Results: Resident to technologist and attending.

\begin{tabular}{|c|c|c|c|}
\hline Total studies performed & Resident & T/A & Overall/p \\
\hline True Positives & 34 & 22 & 462 \\
\hline Sensitivity & 0.59 & 0.65 & 56 \\
\hline $\begin{array}{c}\text { Negative Initial US } \\
\text { Additional studies }\end{array}$ & 216 & 174 & 390 \\
$\begin{array}{c}\text { ordered after a non- } \\
\text { diagnostic US }\end{array}$ & $85(39.5 \%)$ & $46(26.4 \%)$ & $131 / \mathrm{p}=0.01$ \\
\hline False Negatives & $24(41 \%)$ & $11(33 \%)$ & $35 / \mathrm{p}=0.68$ \\
\hline False Positives & 1 & 0 & 1 \\
\hline True Negative & 200 & 167 & 367 \\
\hline Prevalence & 0.23 & 0.17 & 0.2 \\
\hline Equivocal US & $10(3.8 \%)$ & $5(2.5 \%)$ & 15 \\
\hline
\end{tabular}

$\mathrm{T} / \mathrm{A}=$ Technologist And Attending; US = Ultrasound.

( ) Under License of Creative Commons Attribution 3.0 License
Table 2 Pediatric appendicitis ultrasound: Resident performance.

\begin{tabular}{|c|c|c|}
\hline & Total & Results \\
\hline True Positive & 34 & $\begin{array}{l}5 \text { reconfirmed by } \mathrm{CT}, 1 \text { by US, } \\
\text { remaining Sx; } 3 \text { retrocecal, } 2 \text { also } \\
\text { perforated }\end{array}$ \\
\hline False Positive & 1 & Repeat US was negative \\
\hline $\begin{array}{l}\text { Additional studies } \\
\text { ordered after a } \\
\text { negative initial US }\end{array}$ & 85 (of 216 ) & $81 \mathrm{CT}, 2 \mathrm{US}, 2 \mathrm{MR}$ \\
\hline False Negative & 24 & $\begin{array}{l}\text { Confirmed on } 23 \text { CTs, } 1 \text { US; } 10 \\
\text { retrocecal, } 5 \text { perforated ( } 2 \text { also } \\
\text { retrocecal) }\end{array}$ \\
\hline Equivocal Study & 10 & $\begin{array}{l}\text { CT positive in } 2 \text {; both retrocecal, } \\
\text { one also perforated }\end{array}$ \\
\hline
\end{tabular}

US = Ultrasound; Sx = Surgical; CT = Computed Tomography; MR:

Magnetic Resonance.

Table 3 Pediatric appendicitis ultrasound: Technologist and attending performance.

\begin{tabular}{|c|c|c|}
\hline & Total & Results \\
\hline True Positive & 22 & $\begin{array}{l}6 \text { reconfirmed by } C T \text {, remaining } \\
\text { Sx; } 2 \text { retrocecal, } 2 \text { perforated ( } 1 \\
\text { also retrocecal) }\end{array}$ \\
\hline $\begin{array}{l}\text { Additional studies ordered } \\
\text { after a negative initial US }\end{array}$ & $\begin{array}{l}46 \text { (of } \\
174)\end{array}$ & All CTs \\
\hline False Negative & 11 & $\begin{array}{c}11 \text { repeat } \mathrm{CT} ; 8 \text { retrocecal, none } \\
\text { perforated }\end{array}$ \\
\hline Equivocal Study & 5 & $\begin{array}{l}\text { CT positive in } 1 \text {; retrocecal and } \\
\text { perforated }\end{array}$ \\
\hline
\end{tabular}

US = Ultrasound; Sx = Surgical; CT = Computed Tomography

in the TA group, one received a follow up CT, which was positive, with a retrocecal and perforated appendix. Of the initial positive US reports for both groups (56), 7 patients had a retrocecal and/ or perforated appendix. However, in the falsely negative US reports overall (35), there were $23(65.7 \%)$ cases of retrocecal and/or perforated appendices. The overall prevalence for acute appendicitis in our population was $20 \%$.

\section{Discussion}

Ultrasound has traditionally been touted as dependent on the skill of the operator. In our study at a large general urban medical center, the performance of the resident group was on par with the TA group in the proportion of appendicitis correctly diagnosed as well as falsely determined as negative. Our findings suggest that variability in our population may be more a result of limitations of the examination rather than the operator's skill in pediatric appendicitis.

A false-negative US can be due to an aberrant location of the appendix, such as a retrocecal appendix. A multicenter cohort study by M Mittal et al (2013) found variable sensitivity in diagnosing appendicitis across ED sites, in large part due to the varied ability to visualize the appendix [5]. In fact, detection rates of an abnormal appendix vary widely in literature, from $22 \%$ to $98 \%$ [6-8]. A retrocecal appendix can be seen in as much as 20 to $65 \%$ of patients, with intraperitoneal being the most common location $[9,10]$. Given our population this can significantly impact the visualization of the appendix. 
Additional causes of false negative reports can be due to focal (tip) appendicitis or a perforated appendix, which can occur in $20-30 \%$ of the cases [11]. A perforated appendix can make a more difficult diagnosis due to decompression of the appendix lumen. In our study, both groups demonstrated a higher number of retrocecal and/or perforated appendix cases in the false negative US studies while there was a much lower incidence in studies that correctly identified appendicitis on the first US.

A direct comparison to other studies is difficult to make due to lack of a similar comparison group. However the false negative rates for both groups (Table 1) were slightly higher or in line with other studies in the literature [12-14]. We also realize that the reported sensitivity in the literature is higher than that reported in our study, $67 \%$ versus $88 \%$ [15]. However, we believe these differenced to be affected by a higher percentage of obesity unique to our urban population, which can impair the soft tissue penetration of ultrasound and ultimately its sensitivity.

A prior study by Pohl D comparing technologist with and without on site radiologist supervision found little difference in sensitivity and specificity in diagnosing pediatric appendicitis [9]. On the other hand, a study of 1,009 US evaluations in pediatric appendicitis found a higher detection rate of the appendix when the study was performed by specialized pediatric sonographers in comparison to general sonographers; however a conclusion of outcome with regard to appendicitis was not addressed [13]. The only patient specific factor that influenced visualization of the appendix reported was body habitus [13].

Additionally and an original concern of this study, was the statistically significant higher number of additional tests ordered when the initial US study was negative and performed by the resident overnight. Since the majority of the follow up studies were CTs these additional studies result in unnecessary ionizing radiation to the pediatric patients. The results on this study however showed that there was no statistical difference in the prevalence, sensitivity and number of false negatives between the two groups. The additional studies overnight may be a reflection of the clinicians' degree of confidence in a resident performing the exam, or confidence in an ultrasound diagnosis. An alternative explanation can be that the clinicians may have suspected other etiologies in addition to appendicitis for which a CT was additionally ordered. Thus an argument can be made that these additional CTs may have been warranted, although this was not investigated in this study.
A large number of positive cases were also reconfirmed in both groups. It is not clear why this occurred, however it is conceivable that he clinical picture may have been equivocal even while the ultrasound diagnosis was clear. Additionally, given the lack of adequate medical access and delay of care in our patient population, there may be a heightened sensitivity for perforation and abscess by the clinicians, which may not have been seen on ultrasound.

Our study had several limitations. It was not possible to confirm the absence of appendicitis in children who were not operated on, it was assumed these patients did not have appendicitis by lack of further clinical or diagnostic assessment. It is entirely possible that some patients may have had spontaneous resolution of untreated appendicitis or that some patients may have sought care at an outside facility, underestimating the false negatives. Likewise, we must acknowledge that some patients who were highly suspicious for appendicitis may have received surgery without diagnostic imaging. Secondly, this study did not separate residents or attending radiologists by level of experience. Thirdly, this is a single institution study and the results are more applicable to community hospitals. Lastly, we do not know the outcomes of the additional imaging obtained on true negative cases, as alternative diagnoses may have been made warranting such follow up.

We believe that after obtaining minimum competency in the use and interpretation of sonography, diagnosis of appendicitis in a pediatric patient is more likely to be limited due to the aforementioned reasons rather than the experience of the operator in an urban medical center.

\section{Conclusion}

In this study there was no statistical difference between residents of multiple levels of training and technologists and attendings with varying levels of experience in the evaluation of pediatric appendicitis. Additionally, significantly more follow up studies were ordered when the initial US study was negative and performed by the resident resulting in additional ionizing radiation to the pediatric population.

\section{Compliance with ethical standards}

The authors do not have any disclosures or conflicts of interest in this research. For this retrospective study formal consent is not required. This article does not contain any studies with animals performed by any of the authors. 


\section{References}

1 Sivit CJ, Siegel MJ, Applegate KE, Newman KD (2001) When appendicitis is suspected in children. Radiographics 21: 247-62.

2 Rothrock SG, Skeoch G, Rush JJ, Johnson NE (1991) Clinical features of misdiagnosed appendicitis in children. Ann Emerg Med 20: 45-50.

3 Hryhorczuk AL, Mannix RC, Taylor GA (2012) Pediatric abdominal pain: use of imaging in the emergency department in the United States from 1999 to 2007. Radiology.

4 Sivit CJ, Applegate KE (2003) Imaging of acute appendicitis in children. Semin Ultrasound CT-MR 24: 74-82.

5 Mittal MK, Dayan PS, Macias CG, Bachur RG, Bennett J, et al. (2013) Performance of ultrasound in the diagnosis of appendicitis in children in a multicenter cohort. AEM 20: 697-702.

6 Baldisserotto M, Marchiori E (2000) Accuracy of noncompressive sonography of children with appendicitis according to the potential positions of the appendix. Am J Roentgenol 175: 1387-1392.

7 Goldin AB, Khanna P, Thapa M, McBroom JA, Garrison MM, et al. (2011) Revised ultrasound criteria for appendicitis in children improve diagnostic accuracy. Pediatr Radiol 41: 993-999.

8 Garcia Pena BM, Mandl KD, Kraus SJ, Fischer AC, Fleisher GR, et al. (1999) Ultrasonography and limited computed tomography in the diagnosis and management of appendicitis in children. JAMA. 282: 1041-1046.

9 Pohl D, Golub R, Schwartz GE, Stein HD (1998) Appendiceal ultrasonography performed by nonradiologists: does it help in the diagnostic process? J Ultr Med. 17: 217-21.

10 Peletti $A B$, Baldisserotto $M$ (2006) Optimizing US examination to detect the normal and abnormal appendix in children. Pediatr Radiol 36: 1171-6.

11 Quillin SP, Siegel MJ, Coffin CM (1992) Acute appendicitis in children: Value of sonography in detecting perforation. AJR Am J Roentgenol 159: $1265-1268$.

12 WadeDS, MarrowSE, BalsaraZN, BurkhardTK, GoffWB(1993)Accuracy of ultrasound in the diagnosis of acute appendicitis compared with the surgeon's clinical impression. Arch Surg 128:1039-44.

13 Trout AR, Sanchez R, Ladino-Torres MF, Pai DR, Strouse PJ (2012) A critical evaluation of US for the diagnosis of pediatric acute appendicitis in a real-life setting: how can we improve the diagnostic value of sonography? Pediatr Radiol 42: 813-23.

14 Pastore V, Cocomazzi R, Basile A, Pastore M, Bartoli F (2014) Limits and advantages of abdominal ultrasonography in children with acute appendicitis syndrome. Afr J Paediatr Surg. 11: 293-6.

15 Doria AS, Moineddin R, Kellenberger CJ, Epelman M, Beyene, J, et al. (2006) US or CT for diagnosis of appendicitis in children and adults. A Meta-analysis. Radiology 241: 83-94. 